\title{
Knowledge Theoretic Properties of Topological Spaces
}

\author{
Konstantinos Georgatos \\ Department of Mathematics \\ Graduate School and University Center \\ City University of New York \\ 33 West 42nd Street \\ New York, NY 10036
}

\begin{abstract}
We study the topological models of a logic of knowledge for topological reasoning, introduced by Larry Moss and Rohit Parikh (1992). Among our results is a solution of a conjecture by the formentioned authors, finite satisfiability property and decidability for the theory of topological models.
\end{abstract}

\section{Introduction}

We are unable to measure natural quantities with exact precision. Physical devices or bounded resources always introduce a certain amount of error. Because of this fact, we are obliged to limit our observations to approximate values or, better, to sets of possible values. Whenever this happens, sets of points, rather than points, are our subject of reasoning. Thus, the statement "the amount of ozone in the upper atmosphere has decreased by 12 per cent" can never be known to be true with this precision. What we mean is that e.g. the decrease has a value in the interval $(12-\epsilon, 12+\epsilon)$ for some positive real number $\epsilon$. If we are able to spend more resources (taking more samples, using more precise instruments, etc). we may be able to affirm that the value belongs to a smaller interval and therefore to refine our observation. The topology of intervals in the real line is our domain of reasoning.

The above limitations do not prevent us from drawing conclusions. In fact it is enough that we know that a certain quantity belongs to a set of possible values. The first hundred decimal points of $\pi$ are enough for most practical purposes and if we decide to settle for such a value, an algorithm that computes these decimal points conveys the same knowledge as the actual algorithm that computes $\pi$. What we know is exactly the common properties of all algorithms belonging to the same open set of the algorithms we observe in the topology of initial segments and this notion of knowledge coincides with the traditional 
one (Hintikka [1962], Fagin et al. 1991, Halpern and Moses [1984, Parikh and Ramanujam [1985): what is known is whatever is true in all states compatible with the observer's view.

Increase of knowledge is strongly linked with the amount of resources we are willing to invest. An increase of information is accompanied by an increase in the effort of acquiring it. This corresponds to the refinement of the open set in the relevant topology. In the formal system introduced in (Moss and Parikh [1992]) there are two basic modal operators; K for knowledge and $\square$ for effort.

A basic advantage of this logic and its semantics over other temporal logics or logics of change is that, though we make no mention of set, we are able to interpret assertions relative to a set of possible states and, at the same time, keep the dependence on the actual state. Topology is a tool for modelling classes of statements with an intuitionistic flavor such as refutative or affirmative assertions (see (Vickers 1989])) and this logic system enables us to treat them in a classical modal framework. In many respects the way we interpret the modal operator $\mathrm{K}$ resembles the algebraic semantics of a modal operator used to interpret intuitionistic statements as in (Rasiowa and Sikorski [1968). As the intuitionistic version of a statement is the interior of the subset that represents the classical version of it, $\mathrm{K} A$ is satisfied only in the open subsets which are subsets of the interior of the set of points which satisfy a property $A$.

The fundamental reasoning that this logic tries to capture has many equivalents in recursion theory and elsewhere in Mathematics. The discussion of them is well beyond the scope of this paper and the reader is referred to (Georgatos [1993]) and (Moss and Parikh 1992) for a more detailed exposition.

In the following section, we describe the syntax and semantics of the logic and we give complete axiomatisations with respect to subset spaces and topological spaces. In section 3 we develop a theory for describing the validity problem in topological spaces. In section 4 we study the model based on the basis of a topological space closed under finite unions, and we prove it equivalent to the topological space that it generates. These results translate to a completeness theorem for topologies, given a finite axiomatisation for the class of spaces which are closed under (finite) intersection and union. In the last section we prove finite satisfiability for the class of topological models and decidability for their theory.

\section{The logic}

We follow the notation of (Moss and Parikh 1992).

Our language is bimodal and propositional. Formally, we start with a countable set $\mathrm{A}$ of atomic formulae containing two distinguished elements $T$ and $\perp$. Then the language $\mathcal{L}$ is the least set such that $A \subseteq \mathcal{L}$ and closed under the following rules: 


$$
\frac{\phi, \psi \in \mathcal{L}}{\phi \wedge \psi \in \mathcal{L}} \quad \frac{\phi \in \mathcal{L}}{\neg \phi, \square \phi, \mathrm{K} \phi \in \mathcal{L}}
$$

The above language can be interpreted inside any spatial context.

Definition 1 Let $X$ be a set and $\mathcal{O}$ a subset of the powerset of $X$, i.e. $\mathcal{O} \subseteq \mathcal{P}(X)$ such that $X \in \mathcal{O}$. We call the pair $\langle X, \mathcal{O}\rangle$ a subset space. A model is a triple $\langle X, \mathcal{O}, i\rangle$, where $\langle X, \mathcal{O}\rangle$ is a subset space and $i$ a map from A to $\mathcal{P}(X)$ with $i(\top)=X$ and $i(\perp)=\emptyset$ called initial interpretation.

We denote the set $\{(x, U) \mid U \in \mathcal{O}, x \in U\} \subseteq X \times \mathcal{O}$ with $X \dot{\times} \mathcal{O}$. For each $U \in \mathcal{O}$ let $\downarrow U$ be the set $\{V \mid V \in \mathcal{O}$ and $V \subseteq U\}$, i.e. the lower closed set generated by $U$ in the partial order $(\mathcal{O}, \subseteq)$.

Definition 2 The satisfaction relation $\models_{\mathcal{M}}$, where $\mathcal{M}$ is the model $\langle X, \mathcal{O}, i\rangle$, is a subset of $(X \dot{\times} \mathcal{O}) \times \mathcal{L}$ defined recursively by (we write $x, U \models_{\mathcal{M}} \phi$ instead of $\left.((x, U), \phi) \in \models{ }_{\mathcal{M}}\right)$ :

$$
\begin{array}{ll}
x, U \models_{\mathcal{M}} A & \text { iff } \quad x \in i(A), \text { where } A \in \mathrm{A} \\
x, U \models_{\mathcal{M}} \phi \wedge \psi & \text { if } \quad x, U \models_{\mathcal{M}} \phi \text { and } x, U \models_{\mathcal{M}} \psi \\
x, U \models_{\mathcal{M}} \neg \phi & \text { if } \quad x, U \models_{\mathcal{M}} \phi \\
x, U \models_{\mathcal{M}} \mathrm{K} \phi & \text { if } \quad \text { for all } y \in U, \quad y, U \models_{\mathcal{M}} \phi \\
x, U \models_{\mathcal{M}} \square \phi & \text { if } \quad \text { for all } V \in \downarrow U \text { such that } x \in V, \quad x, V \models_{\mathcal{M}} \phi .
\end{array}
$$

If $x, U \models{ }_{\mathcal{M}} \phi$ for all $(x, U)$ belonging to $X \dot{\times} \mathcal{O}$ then $\phi$ is valid in $\mathcal{M}$, denoted by $\mathcal{M} \models \phi$.

We abbreviate $\neg \square \neg \phi$ and $\neg \mathrm{K} \neg \phi$ with $\diamond \phi$ and $\mathrm{L} \phi$ respectively. We have that

$x, U \models_{\mathcal{M}} \mathrm{L} \phi \quad$ if there exists $y \in U$ such that $y, U=_{\mathcal{M}} \phi$ and,

$x, U \models{ }_{\mathcal{M}} \diamond \phi \quad$ if there exists $V \in \mathcal{O}$ such that $V \subseteq U, x \in V$, and $x, V \models{ }_{\mathcal{M}} \phi$.

Many topological properties are expressible in this logical system in a natural way. For instance, in a model where the subset space is a topological space, $i(A)$ is open whenever $A \rightarrow \diamond \mathrm{K} A$ is valid in this model or $i(A)$ is nowhere dense whenever $\mathrm{L} \diamond \mathrm{K} \neg A$ is valid (cf. (Moss and Parikh 1992)).

Example 3 Consider the set of real numbers $\mathbf{R}$ with the usual topology of open intervals. We define the following three predicates:

$$
\begin{array}{rll}
\text { pi } & \text { where } & i(\mathrm{pi})=\{\pi\} \\
\mathrm{I}_{1} & \text { where } & i\left(\mathrm{I}_{1}\right)=(-\infty, \pi] \\
\mathrm{I}_{2} & \text { where } & i\left(\mathrm{I}_{2}\right)=(\pi,+\infty) \\
\mathrm{Q} & \text { where } & i(\mathrm{Q})=\{q \mid q \text { is rational }\} .
\end{array}
$$


There is no real number $p$ and open set $U$ such that $p, U \models \mathrm{Kpi}$ because that would imply $p=\pi$ and $U=\{\pi\}$ and there are no singletons which are open.

A point $x$ belongs to the closure of a set $W$ if every open $U$ that contains $x$ intersects $W$. Thus $\pi$ belongs to the closure of $(\pi,+\infty)$, i.e every open that contains $\pi$ has a point in $(\pi,+\infty)$. This means that for all $U$ such that $\pi \in U$, $\pi, U \models \mathrm{LI}_{2}$, therefore $\pi, \mathbf{R} \models \square \mathrm{LI}_{2}$. Following the same reasoning $\pi, \mathbf{R} \models \square \mathrm{LI}_{1}$, since $\pi$ belongs to the closure of $(-\infty, \pi]$.

A point $x$ belongs to the boundary of a set $W$ whenever $x$ belong to the closure of $W$ and $X-W$. By the above, $\pi$ belongs to the boundary of $(-\infty, \pi]$ and $\pi, \mathbf{R} \models \square\left(\mathrm{LI}_{1} \wedge \mathrm{LI}_{2}\right)$.

A set $W$ is closed if it contains its closure. The interval $i\left(\mathrm{I}_{1}\right)=(-\infty, \pi]$ is closed and this means that the formula $\square \mathrm{LI}_{1} \rightarrow \mathrm{I}_{1}$ is valid.

A set $W$ is dense if all opens contain a point of $W$. The set of rational numbers is dense which translates to the fact that the formula $\square \mathrm{LQ}$ is valid. To exhibit the reasoning in this logic, suppose that the set of rational numbers was closed then both $\square \mathrm{LQ}$ and $\square \mathrm{LQ} \rightarrow \mathrm{Q}$ would be valid. This implies that Q would be valid which means that all reals would be rationals. Hence the set of rational numbers is not closed.

The following set of axioms and rules, denoted by $\mathbf{M P}^{*}$, is sound and complete for the class of topological spaces (see (Georgatos 1993)) while axioms 1 through 10, denoted by MP, appeared first and proven sound and complete for the class of subset spaces in (Moss and Parikh 1992).

\section{Axioms}

1. All propositional tautologies

2. $(A \rightarrow \square A) \wedge(\neg A \rightarrow \square \neg A)$, for $A \in \mathrm{A}$

3. $\square(\phi \rightarrow \psi) \rightarrow(\square \phi \rightarrow \square \psi)$

4. $\square \phi \rightarrow \phi$

5. $\square \phi \rightarrow \square \square \phi$

6. $\mathrm{K}(\phi \rightarrow \psi) \rightarrow(\mathrm{K} \phi \rightarrow \mathrm{K} \psi)$

7. $\mathrm{K} \phi \rightarrow \phi$

8. $\mathrm{K} \phi \rightarrow \mathrm{KK} \phi$

9. $\phi \rightarrow \mathrm{KL} \phi$

10. $\mathrm{K} \square \phi \rightarrow \square \mathrm{K} \phi$

11. $\diamond \square \phi \rightarrow \square \diamond \phi$

12. $\diamond(\mathrm{K} \phi \wedge \psi) \wedge \mathrm{L} \diamond(\mathrm{K} \phi \wedge \chi) \rightarrow \diamond(\mathrm{K} \diamond \phi \wedge \diamond \psi \wedge \mathrm{L} \diamond \chi)$ 


\section{Rules}

$$
\begin{gathered}
\frac{\phi \rightarrow \psi, \phi}{\psi} \text { MP } \\
\frac{\phi}{\mathrm{K} \phi} \text { K-Necessitation } \frac{\phi}{\square \phi} \text { 口-Necessitation }
\end{gathered}
$$

\section{$3 \quad$ Stability and Splittings}

Suppose that $X$ is a set and $\mathcal{T}$ a topology on $X$. In the following we assume that we are working in the topological space $(X, \mathcal{T})$. Our aim is to find a partition of $\mathcal{T}$, where a given formula $\phi$ "retains its truth value" for each point throughout a member of this partition. We shall show that there exists a finite partition of this kind.

Definition 4 Given a finite family $\mathcal{F}=\left\{U_{1}, \ldots, U_{n}\right\}$ of opens, we define the remainder of (the principal ideal in $\left(\mathcal{T}, \subseteq\right.$ ) generated by) $U_{k}$ by

$$
\operatorname{Rem}^{\mathcal{F}} U_{k}=\downarrow U_{k}-\bigcup_{U_{k} \nsubseteq U_{i}} \downarrow U_{i}
$$

Proposition 5 In a finite set of opens $\mathcal{F}=\left\{U_{1}, \ldots, U_{n}\right\}$ closed under intersection, we have

$$
\operatorname{Rem}^{\mathcal{F}} U_{i}=\downarrow U_{i}-\bigcup_{U_{j} \subset U_{i}} \downarrow U_{j}
$$

for $i=1, \ldots, n$.

Proof.

$$
\begin{aligned}
\operatorname{Rem}^{\mathcal{F}} U_{i} & =\downarrow U_{i}-\bigcup_{U_{i} \nsubseteq U_{h}} \downarrow U_{h} \\
& =\downarrow U_{i}-\bigcup_{U_{i} \nsubseteq U_{h}} \downarrow\left(U_{h} \cap U_{i}\right) \\
& =\downarrow U_{i}-\bigcup_{U_{j} \subset U_{i}} \downarrow U_{i} .
\end{aligned}
$$

We denote $\bigcup_{U_{i} \in \mathcal{F}} \downarrow U_{i}$ with $\downarrow \mathcal{F}$.

Proposition 6 If $\mathcal{F}=\left\{U_{1}, \ldots, U_{n}\right\}$ is a finite family of opens, closed under intersection, then

a. $\operatorname{Rem}^{\mathcal{F}} U_{i} \cap \operatorname{Rem}^{\mathcal{F}} U_{j}=\emptyset$, for $i \neq j$,

b. $\bigcup_{i=1}^{n} \operatorname{Rem}^{\mathcal{F}} U_{i}=\downarrow \mathcal{F}$, i.e. $\left\{\operatorname{Rem}^{\mathcal{F}} U_{i}\right\}_{i=1}^{n}$ is a partition of $\downarrow \mathcal{F}$. We call such an $\mathcal{F}$ a finite splitting (of $\downarrow \mathcal{F}$ ),

c. if $V_{1}, V_{3} \in \operatorname{Rem}^{\mathcal{F}} U_{i}$ and $V_{2}$ is an open such that $V_{1} \subseteq V_{2} \subseteq V_{3}$ then $V_{2} \in \operatorname{Rem}^{\mathcal{F}} U_{i}$, i.e. $\operatorname{Rem}^{\mathcal{F}} U_{i}$ is convex.

Proof. The first and the third are immediate from the definition.

For the second, suppose that $V \in \downarrow \mathcal{F}$ then $V \in \operatorname{Rem}^{\mathcal{F}} \bigcap_{V \in \downarrow U_{i}} U_{i}$. 
Every partition of a set induces an equivalence relation on this set. The members of the partition comprise the equivalence classes. Since a splitting induces a partition, we denote the equivalence relation induced by a splitting $\mathcal{F}$ by $\sim_{\mathcal{F}}$.

Definition 7 Given a set of open subsets $\mathcal{G}$, we define the relation $\sim_{\mathcal{G}}^{\prime}$ on $\mathcal{T}$ with $V_{1} \sim_{\mathcal{G}}^{\prime} V_{2}$ if and only if $V_{1} \subseteq U \Leftrightarrow V_{2} \subseteq U$ for all $U \in \mathcal{G}$.

We have the following

Proposition 8 The relation $\sim_{\mathcal{G}}^{\prime}$ is an equivalence.

Proposition 9 Given a finite splitting $\mathcal{F}, \sim_{\mathcal{F}}^{\prime}=\sim_{\mathcal{F}}$ i.e. the remainders of $\mathcal{F}$ are the equivalence classes of $\sim_{\mathcal{F}}^{\prime}$.

Proof. Suppose $V_{1} \sim_{\mathcal{F}}^{\prime} V_{2}$ then $V_{1}, V_{2} \in \operatorname{Rem}^{\mathcal{F}} U$, where

$$
U=\bigcap\left\{U^{\prime} \mid V_{1}, V_{2} \subseteq U, U^{\prime} \in \mathcal{F}\right\} .
$$

For the other way suppose $V_{1}, V_{2} \in \operatorname{Rem}^{\mathcal{F}} U$ and that there exists $U^{\prime} \in \mathcal{F}$ such that $V_{1} \subseteq U^{\prime}$ while $V_{2} \not \subset U^{\prime}$. Then we have that $V_{1} \subseteq U^{\prime} \cap U, U^{\prime} \cap U \in \mathcal{F}$ and $U^{\prime} \cap U \subseteq U$ i.e. $V_{1} \notin \operatorname{Rem}^{\mathcal{F}} U$.

We state some useful facts about splittings.

Proposition 10 If $\mathcal{G}$ is a finite set of opens, then $\mathrm{Cl}(\mathcal{G})$, its closure under intersection, yields a finite splitting for $\downarrow \mathcal{G}$.

The last proposition enables us to give yet another characterization of remainders: every family of points in a complete lattice closed under arbitrary joins comprises a closure system, i.e. a set of fixed points of a closure operator of the lattice (cf. (Gierz et al. 1980)). Here, the lattice is the poset of the opens of the topological space. If we restrict ourselves to a finite number of fixed points then we just ask for a finite set of opens closed under intersection i.e. Proposition 10. Thus a closure operator in the lattice of the open subsets of a topological space induces an equivalence relation, two opens being equivalent if they have the same closure, and the equivalence classes of this relation are just the remainders of the open subsets which are fixed points of the closure operator. The maximum open in $\operatorname{Rem}^{\mathcal{F}} U$, i.e. $U$, can be taken as the representative of the equivalence class which is the union of all open sets belonging to $\operatorname{Rem}^{\mathcal{F}} U$.

We now introduce the notion of stability corresponding to what we mean by "a formula retains its truth value on a set of opens".

Definition 11 If $\mathcal{G}$ is a set of opens then $\mathcal{G}$ is stable for $\phi$, if for all $x$, either $x, V \models \phi$ for all $V \in \mathcal{G}$, or $x, V \models \neg \phi$ for all $V \in \mathcal{G}$, such that $x \in V$.

Proposition 12 If $\mathcal{G}_{1}, \mathcal{G}_{2}$ are sets of opens then 
a. if $\mathcal{G}_{1} \subseteq \mathcal{G}_{2}$ and $\mathcal{G}_{2}$ is stable for $\phi$ then $\mathcal{G}_{1}$ is stable for $\phi$,

b. if $\mathcal{G}_{1}$ is stable for $\phi$ and $\mathcal{G}$ is stable for $\chi$ then $\mathcal{G}_{1} \cap \mathcal{G}_{2}$ is stable for $\phi \wedge \chi$.

Proof. (国) is easy to see while (b) is a corollary of (a).

Definition 13 A finite splitting $\mathcal{F}=\left\{U_{1}, \ldots, U_{n}\right\}$ is called a stable splitting for $\phi$, if $\operatorname{Rem}^{\mathcal{F}} U_{i}$ is stable for $\phi$ for all $U_{i} \in \mathcal{F}$.

Proposition 14 If $\mathcal{F}=\left\{U_{1}, \ldots, U_{n}\right\}$ is a stable splitting for $\phi$, so is

$$
\mathcal{F}^{\prime}=\mathrm{Cl}\left(\left\{U_{0}, U_{1}, \ldots, U_{n}\right\}\right),
$$

where $U_{0} \in \downarrow \mathcal{F}$.

Proof. Let $V \in \mathcal{F}^{\prime}$ then there exists $U_{l} \in \mathcal{F}$ such that $\operatorname{Rem}^{\mathcal{F}^{\prime}} V \subseteq \operatorname{Rem}^{\mathcal{F}} U_{l}$ (e.g. $\left.U_{l}=\bigcap\left\{U_{i} \mid U_{i} \in \mathcal{F}, V \subseteq U_{i}\right\}\right)$ i.e. $\mathcal{F}^{\prime}$ is a refinement of $\mathcal{F}$. But $\operatorname{Rem}^{\mathcal{F}} U_{l}$ is stable for $\phi$ and so is $\operatorname{Rem}^{\mathcal{F}^{\prime}} V$ by Proposition 12(2).

The above proposition tells us that if there is a finite stable splitting for a topology then there is a closure operator with finitely many fixed points whose associated equivalence classes are stable sets of open subsets.

Suppose that $\mathcal{M}=\langle X, \mathcal{T}, i\rangle$ is a topological model for $\mathcal{L}$. Let $\mathcal{F}_{\mathcal{M}}$ be a family of subsets of $X$ generated as follows: $i(A) \in \mathcal{F}_{\mathcal{M}}$ for all $A \in \mathrm{A}$, if $S \in \mathcal{F}_{\mathcal{M}}$ then $X-S \in \mathcal{F}_{\mathcal{M}}$, if $S, T \in \mathcal{F}_{\mathcal{M}}$ then $S \cap T \in \mathcal{F}_{\mathcal{M}}$, and if $S \in \mathcal{F}_{\mathcal{M}}$ then $S^{\circ} \in \mathcal{F}_{\mathcal{M}}$ i.e. $\mathcal{F}_{\mathcal{M}}$ is the least set containing $\{i(A) \mid A \in \mathrm{A}\}$ and closed under complements, intersections and interiors. Let $\mathcal{F}_{\mathcal{M}}^{\circ}$ be the set $\left\{S^{\circ} \mid S \in \mathcal{F}_{\mathcal{M}}\right\}$. We have $\mathcal{F}_{\mathcal{M}}^{\circ}=\mathcal{F}_{\mathcal{M}} \cap \mathcal{T}$. The following is the main theorem of this section.

Theorem 15 (Partition Theorem) Let $\mathcal{M}=\langle X, \mathcal{T}, i\rangle$ be a topological model. Then there exists a set $\left\{\mathcal{F}^{\psi}\right\}_{\psi \in \mathcal{L}}$ of finite stable splittings such that

1. $\mathcal{F}^{\psi} \subseteq \mathcal{F}_{\mathcal{M}}^{\circ}$ and $X \in \mathcal{F}^{\psi}$, for all $\psi \in \mathcal{L}$,

2. if $U \in \mathcal{F}^{\psi}$ then $U^{\psi}=\{x \in U \mid x, U \models \psi\} \in \mathcal{F}_{\mathcal{M}}$, and

3. if $\phi$ is a subformula of $\psi$ then $\mathcal{F}^{\phi} \subseteq \mathcal{F}^{\psi}$ and $\mathcal{F}^{\psi}$ is a finite stable splitting for $\phi$,

where $\mathcal{F}_{\mathcal{M}}, \mathcal{F}_{\mathcal{M}}^{\circ}$ as above.

Proof. By induction on the structure of the formula $\psi$. In each step we take care to refine the partition of the induction hypothesis.

- If $\psi=A$ is an atomic formula, then $\mathcal{F}^{A}=\{X, \emptyset\}=\{i(\top), i(\perp)\}$, since $\mathcal{T}$ is stable for all atomic formulae. We also have $\mathcal{F}^{A} \subseteq \mathcal{F}_{\mathcal{M}}^{\circ}$ and $X^{A}=$ $i(A) \in \mathcal{F}_{\mathcal{M}}$. 
- If $\psi=\neg \phi$ then let $\mathcal{F}^{\psi}=\mathcal{F}^{\phi}$, since the statement of the proposition is symmetric with respect to negation. We also have that for an arbitrary $U \in \mathcal{F}^{\psi}, U^{\psi}=U^{\urcorner} \phi$.

- If $\psi=\chi \wedge \phi$, let

$$
\mathcal{F}^{\psi}=\mathrm{Cl}\left(\mathcal{F}^{\chi} \cup \mathcal{F}^{\phi}\right) .
$$

Observe that $\mathcal{F}^{\chi} \cup \mathcal{F}^{\phi} \subseteq \mathcal{F}^{\chi \wedge \phi}$.

Now, if $W_{i} \in \mathcal{F}^{\psi}$ then there exists $U_{j} \in \mathcal{F}^{\chi}$ and $V_{k} \in \mathcal{F}^{\phi}$ such that

$$
W_{i}=U_{j} \cap V_{k} \quad \text { and } \quad \operatorname{Rem}^{\mathcal{F}^{\psi}} W_{i} \subseteq \operatorname{Rem}^{\mathcal{F} \chi} U_{j} \cap \operatorname{Rem}^{\mathcal{F}^{\phi}} V_{k}
$$

(e.g. $U_{j}=\bigcap\left\{U_{m} \mid W_{i} \subseteq U_{m}, U_{m} \in \mathcal{F}^{\chi}\right\}$ and $V_{k}=\bigcap\left\{V_{n} \mid W_{i} \subseteq\right.$ $\left.V_{n}, V_{n} \in \mathcal{F}^{\phi}\right\}$ ). Since $\operatorname{Rem}^{\mathcal{F}^{\chi}} U_{j}$ is stable for $\chi$ and $\operatorname{Rem}^{\mathcal{F}^{\phi}} V_{n}$ is stable for $\phi$, their intersection is stable for $\chi \wedge \phi=\psi$, by Proposition 12(B), and so is its subset $\operatorname{Rem}^{\mathcal{F}^{\psi}} W_{i}$, by Proposition 12(可). Thus $\mathcal{F}^{\psi}$ is a finite stable splitting for $\psi$ containing $X$.

We have that $\mathcal{F}^{\psi} \subseteq \mathcal{F}_{\mathcal{M}}$ whenever $\mathcal{F}^{\chi} \subseteq \mathcal{F}_{\mathcal{M}}$ and $\mathcal{F}^{\phi} \subseteq \mathcal{F}_{\mathcal{M}}^{\circ}$. Finally, $W_{i}^{\psi}=U_{j}^{\chi} \cap V_{k}^{\phi}$.

- Suppose $\psi=\mathrm{K} \phi$. Then, by induction hypothesis, there exists a finite stable splitting $\mathcal{F}^{\phi}=\left\{U_{1}, \ldots, U_{n}\right\}$ for $\phi$ containing $X$. Let

$$
W_{i}=\left(U_{i}^{\phi}\right)^{\circ}
$$

for all $i \in\{1, \ldots, n\}$.

Observe that if $x \in U_{i}-W_{i}$ then $x, V \models \neg \phi$, for all $V \in \operatorname{Rem}^{\mathcal{F}^{\phi}} U_{i}$ and $x \in V$, since $\operatorname{Rem}^{\mathcal{F}^{\phi}} U_{i}$ is stable for $\phi$, by induction hypothesis.

Now, if $V \in \operatorname{Rem}^{\mathcal{F}^{\phi}} U_{i} \cap \downarrow W_{i}$, for some $i \in\{1 \ldots, n\}$, then $x, V \models \phi$ for all $x \in V$, by definition of $W_{i}$, hence $x, V \models \mathrm{K} \phi$ for all $x \in V$.

On the other hand, if $V \in \operatorname{Rem}^{\mathcal{F}^{\phi}} U_{i}-\downarrow W_{i}$ then there exists $x \in V$ such that $x, V \models \neg \phi$ (otherwise $V \subseteq W_{i}$ ). Thus we have $x, V \models \neg \mathrm{K} \phi$ for all $x \in V$. Hence $\operatorname{Rem}^{\mathcal{F}^{\phi}} U_{i} \cap \downarrow W_{i}$ and $\operatorname{Rem}^{\mathcal{F}^{\phi}} U_{i}-\downarrow W_{i}$ are stable for $\mathrm{K} \phi$. Thus, the set

$F=\left\{\operatorname{Rem}^{\mathcal{F}} U_{i} \mid W_{i} \notin \operatorname{Rem}^{\mathcal{F}} U_{i}\right\} \cup\left\{\operatorname{Rem}^{\mathcal{F}} U_{j}-\downarrow W_{j}, \operatorname{Rem}^{\mathcal{F}} U_{j} \cap \downarrow W_{j} \mid W_{j} \in U_{j}\right\}$

is a partition of $\mathcal{T}$ and its members are stable for $\mathrm{K} \phi$. Let $\sim_{F}$ be the equivalence relation on $\mathcal{T}$ induced by $F$ and let

$$
\mathcal{F}^{\mathrm{K} \phi}=\operatorname{Cl}\left(\mathcal{F}^{\phi} \cup\left\{W_{i} \mid W_{i} \in \operatorname{Rem}^{\mathcal{F}^{\phi}} U_{i}\right\}\right) .
$$

We have that $\mathcal{F}^{\mathrm{K} \phi}$ is a finite set of opens and $\mathcal{F}^{\phi} \subseteq \mathcal{F}^{\mathrm{K} \phi}$. Thus, $\mathcal{F}^{\mathrm{K} \phi}$ is finite and contains $X$. We have only to prove that $\mathcal{F}^{\overline{\mathrm{K}}} \phi$ is a stable splitting for $\mathrm{K} \phi$, i.e. every remainder of an open in $\mathcal{F}^{\mathrm{K} \phi}$ is stable for $\mathrm{K} \phi$. 
If $V_{1} \chi_{F} V_{2}$, where $V_{1}, V_{2} \in \mathcal{T}$, then there exists $U=U_{i}$ or $W_{i}$ for some $i=1, \ldots, n$ such that $V_{1} \subseteq U$ while $V_{2} \nsubseteq U$. But this implies that $V_{1} \chi_{\mathcal{F} \mathrm{K}_{\phi}} V_{2}$. Therefore $\left\{\operatorname{Rem}^{\mathcal{F}^{\mathrm{K}} \phi} U\right\}_{U \in \mathcal{F}} \mathrm{K}_{\phi}$ is a refinement of $F$ and $\mathcal{F}^{\mathrm{K} \phi}$ is a finite stable splitting for $\mathrm{K} \phi$ using Proposition 12(2).

We have that $\mathcal{F}^{\mathrm{K} \phi} \subseteq \mathcal{F}_{\mathcal{M}}^{\circ}$ because $W_{i} \in \mathcal{F}_{\mathcal{M}}^{\circ}$, for $i=1, \ldots, n$. Now if $U \in \mathcal{F}^{\psi}$ then either $U^{\mathrm{K} \phi}=U$ or $U^{\mathrm{K} \phi}=\emptyset$.

- Suppose $\psi=\square \phi$. Then, by induction hypothesis, there exists a finite stable splitting $\mathcal{F}^{\phi}=\left\{U_{1}, \ldots, U_{n}\right\}$ for $\phi$ containing $X$.

Let

$$
\mathcal{F}^{\square \phi}=\mathrm{Cl}\left(\mathcal{F}^{\phi} \cup\left\{U_{i} \Rightarrow U_{j} \mid 1 \leq i, j \leq n\right\}\right),
$$

where $\Rightarrow$ is the implication of the complete Heyting algebra $\mathcal{T}$ i.e. $V \subseteq$ $U \Rightarrow W$ if and only if $V \cap U \subseteq W$ for $V, U, W \in \mathcal{T}$. We have that $U \Rightarrow W$ equals $(X-(U-W))^{\circ}$. Clearly, $\mathcal{F}^{\square \phi}$ is a finite splitting containing $X$ and $\mathcal{F}^{\phi} \subseteq \mathcal{F}^{\square \phi}$. We have only to prove that $\mathcal{F}^{\square \phi}$ is stable for $\square \phi$. But first, we prove the following claim:

Claim 16 Suppose $U \in \mathcal{F}^{\phi}$ and $U^{\prime} \in \mathcal{F}^{\square \phi}$. Then

$$
U^{\prime} \cap U \in \operatorname{Rem}^{\mathcal{F}^{\phi}} U \Longleftrightarrow V \cap U \in \operatorname{Rem}^{\mathcal{F}^{\phi}} U \text { for all } V \in \operatorname{Rem}^{\mathcal{F}^{\square \phi}} U^{\prime} .
$$

Proof. The one direction is straightforward. For the other, let $V \in$ $\operatorname{Rem}^{\mathcal{F}^{\square \phi}} U^{\prime}$ and suppose $V \cap U \notin \operatorname{Rem}^{\mathcal{F}^{\phi}} U$ towards a contradiction. This implies that there exists $U^{\prime \prime} \in \mathcal{F}^{\phi}$, with $U^{\prime \prime} \subset U$, such that $V \cap U \subseteq U^{\prime \prime}$. Thus, $V \subseteq U \Rightarrow U^{\prime \prime}$ but $U^{\prime} \nsubseteq U \Rightarrow U^{\prime \prime}$. But $U \Rightarrow U^{\prime \prime} \in \mathcal{F}^{\square \phi}$ which contradicts $U^{\prime} \sim_{\mathcal{F}^{\square} \phi} V$, by Proposition 9 .

Let $U^{\prime} \in \mathcal{F}^{\square \phi}$. We must prove that $\operatorname{Rem}^{\mathcal{F}^{\square \phi}} U^{\prime}$ is stable for $\square \phi$.

Suppose that $x, U^{\prime} \models \neg \square \phi$. We must prove that

$$
x, V^{\prime} \models \neg \square \phi
$$

for all $V^{\prime} \in \operatorname{Rem}^{\mathcal{F}^{\square \phi}} U^{\prime}$ such that $x \in V^{\prime}$.

Since $x, U^{\prime} \models \neg \square \phi$, there exists $V \in \mathcal{T}$, with $x \in V$ and $V \subseteq U^{\prime}$, such that $x, V \models \neg \phi$. Since $\mathcal{F}^{\phi}$ is a splitting, there exists $U \in \mathcal{F}^{\phi}$ such that $V \in \operatorname{Rem}^{\mathcal{F}^{\phi}} U$. Observe that $V \subseteq U^{\prime} \cap U \subseteq U$, so $U^{\prime} \cap U \in \operatorname{Rem}^{\mathcal{F}^{\phi}} U$, by Proposition 6(c).

By Claim 16, for all $V^{\prime} \in \operatorname{Rem}^{\mathcal{F}^{\square \phi}} U^{\prime}$, we have $V^{\prime} \cap U \in \operatorname{Rem}^{\mathcal{F}^{\phi}} U$. Thus if $x \in V^{\prime}$ then $x, V^{\prime} \cap U \models \neg \phi$, because $\operatorname{Rem}^{\mathcal{F}^{\phi}} U$ is stable for $\phi$ by induction hypothesis. This implies that, for all $V^{\prime}$ such that $V^{\prime} \in \operatorname{Rem}^{\mathcal{F}^{\square \phi}} U^{\prime}$ and $x \in V$, we have $x, V^{\prime} \models \neg \square \phi$. 
Therefore, $\mathcal{F}^{\square \phi}$ is a finite stable splitting for $\square \phi$.

Now $U_{i} \Rightarrow U_{j} \in \mathcal{F}_{\mathcal{M}}^{\circ}$ for $1 \leq i, j \leq n$, hence $\mathcal{F}^{\square \phi} \subseteq \mathcal{F}_{\mathcal{M}}^{\circ}$.

Finally, let $U$ belong to $\mathcal{F}^{\square \phi}$ and $V_{1}, \ldots, V_{m}$ be all opens in $\mathcal{F}^{\phi}$ such that $U \cap V_{i} \in \operatorname{Rem}^{\mathcal{F}^{\phi}} V_{i}$, for $i=1, \ldots, m$. Then $x, U \models \diamond \neg \phi$ if and only if there exists $j \in\{1, \ldots, m\}$ with $x \in V_{j}$ and $x, V_{j} \models \neg \phi$ because $x, V_{j} \cap U \models \neg \phi$ since $V_{j} \cap U \in \operatorname{Rem}^{\mathcal{F}^{\phi}} V_{j}$. This implies that

$$
U^{\neg \square \phi}=U^{\diamond \neg \phi}=U \cap \bigcup_{i=1}^{m} V_{i}^{\neg \phi} .
$$

Since $U, V_{1}^{\neg \phi}, \ldots, V_{m}^{\neg \phi}$ belong to $\mathcal{F}_{\mathcal{M}}$, so does $U^{\neg \square \phi}$ and, therefore, $U^{\square \phi}=$ $U-U \neg \square \phi$.

In all steps of induction we refine the finite splitting, so if $\phi$ is a subformula of $\psi$ then $\mathcal{F}^{\phi} \subseteq \mathcal{F}^{\psi}$ and $\mathcal{F}^{\psi}$ is stable for $\phi$ using Proposition 12(国).

Theorem 15 gives us a great deal of intuition for topological models. It describes in detail the expressible part of the topological lattice for the completeness result as it appears in (Georgatos 1993) and paves the road for the reduction of the theory of topological models to that of spatial lattices and the decidability result of this section.

\section{Basis Model}

Let $\mathcal{T}$ be a topology on a set $X$ and $\mathcal{B}$ a basis for $\mathcal{T}$. We denote satisfaction in the models $\langle X, \mathcal{T}, i\rangle$ and $\langle X, \mathcal{B}, i\rangle$ by $\models_{\mathcal{T}}$ and $\models_{\mathcal{B}}$, respectively. In the following proposition we prove that each equivalence class under $\sim_{\mathcal{F}}$ contains an element of a basis closed under finite unions.

Proposition 17 Let $(X, \mathcal{T})$ be a topological space, and let $\mathcal{B}$ be a basis for $\mathcal{T}$ closed under finite unions. Let $\mathcal{F}$ be any finite subset of $\mathcal{T}$. Then for all $V \in \mathcal{F}$ and all $x \in V$, there is some $U \in \mathcal{B}$ with $x \in U \subseteq V$ and $U \in \operatorname{Rem}^{\mathcal{F}} V$.

Proof. By finiteness of $\mathcal{F}$, let $V_{1}, \ldots, V_{k}$ be the elements of $\mathcal{F}$ such that $V \nsubseteq V_{i}$, for $i \in\{1, \ldots, k\}$. Since $V_{i} \neq V$, take $x_{i} \in V-V_{i}$ for $i \in\{1, \ldots, k\}$. Since $\mathcal{B}$ is a basis for $\mathcal{T}$, there exist $U_{x}, U_{i}$, with $x \in U_{x}$ and $x_{i} \in U_{i}$, such that $U_{x}$ and $U_{i}$ are subsets of $V$ for $i \in\{1, \ldots, k\}$. Set

$$
U=\left(\bigcup_{i=1}^{k} U_{i}\right) \cup U_{x}
$$

Observe that $x \in U$, and $U \in \mathcal{B}$, as it is a finite union of members of $\mathcal{B}$. Also $U \in \operatorname{Rem}^{\mathcal{F}} V$, since $U \in \downarrow V$ but $U \notin \bigcup \downarrow V_{i}$ for $i \in\{1, \ldots, k\}$. 
Corollary 18 Let $(X, \mathcal{T})$ be a topological space, $\mathcal{B}$ a basis for $\mathcal{T}$ closed under finite unions, $x \in X$ and $U \in \mathcal{B}$. Then

$$
x, U \models_{\mathcal{T}} \phi \quad \Longleftrightarrow \quad x, U \models_{\mathcal{B}} \phi .
$$

Proof. By induction on $\phi$.

The interesting case is when $\phi=\square \psi$. Fix $x, U$, and $\psi$. By Proposition 15, there exists a finite stable splitting $\mathcal{F}$ for $\phi$ and its subformulae such that $\mathcal{F}$ contains $X$ and $U$. Assume that $x, U \models_{\mathcal{B}^{B}} \square \psi$, and $V \in \mathcal{T}$ such that $V \subseteq U$. By Proposition 6(b), there is some $V^{\prime} \subseteq U$ in $\mathcal{F}$ with $V \in \operatorname{Rem}^{\mathcal{F}} V^{\prime}$. By Proposition 17, let $W \in \mathcal{B}$ be such that $W \in \operatorname{Rem}^{\mathcal{F}} V^{\prime}$ with $x \in W$. So $x, W \models_{\mathcal{B}} \psi$, and thus by induction hypothesis, $x, W \models_{\mathcal{T}} \psi$. By stability, twice, $x, V \models_{\mathcal{T}} \psi$ as well.

We are now going to prove that a model based on a topological space $\mathcal{T}$ is equivalent to the one induced by any basis of $\mathcal{T}$ which is lattice. Observe that this enables us to reduce the theory of topological spaces to that of spatial lattices and, therefore, to answer the conjecture of (Moss and Parikh 1992) : a completeness theorem for subset spaces which are lattices will extend to the smaller class of topological spaces.

Theorem 19 Let $(X, \mathcal{T})$ be a topological space and $\mathcal{B}$ a basis for $\mathcal{T}$ closed under finite unions. Let $\mathcal{M}_{1}=\langle X, \mathcal{T}, i\rangle$ and $\mathcal{M}_{2}=\langle X, \mathcal{B}, i\rangle$ be the corresponding models. Then, for all $\phi$,

$$
\mathcal{M}_{1} \models \phi \quad \Longleftrightarrow \quad \mathcal{M}_{2} \models \phi
$$

Proof. It suffices to prove that $x, U \models_{\mathcal{T}} \phi$ for some $U \in \mathcal{T}$, if and only if $x, U^{\prime} \models_{\mathcal{B}} \phi$ for some $U^{\prime} \in \mathcal{B}$.

Suppose $x, U \models_{\mathcal{T}} \phi$, where $U \in \mathcal{T}$, then, by Corollary 18 , there exists $U^{\prime} \in \mathcal{B}$ such that $x \in U^{\prime}$ and $x, U \models_{\mathcal{T}} \phi$. By Corollary 18, $x, U^{\prime} \models_{\mathcal{B}} \phi$.

Suppose $x, U \models_{\mathcal{B}} \phi$, where $U \in \mathcal{B}$, then $x, U \models_{\mathcal{T}} \phi$, by Corollary 18 .

\section{$5 \quad$ Finite Satisfiability}

Proposition 20 Let $\langle X, \mathcal{T}\rangle$ be a subset space. Let $\mathcal{F}$ be a finite stable splitting for a formula $\phi$ and all its subformulae, and assume that $X \in \mathcal{F}$. Then for all $U \in \mathcal{F}$, all $x \in U$, and all subformulae $\psi$ of $\phi, x, U \models_{\mathcal{T}} \psi$ iff $x, U \models_{\mathcal{F}_{\mathcal{F}}} \psi$.

Proof. The argument is by induction on $\phi$. The only interesting case to consider is when $\phi=\square \psi$. 
Suppose first that $x, U \models_{\mathcal{F}} \square \psi$ with $U \in \mathcal{F}$. We must show that $x, U \models_{\mathcal{T}} \square \psi$ also. Let $V \in \mathcal{T}$ such that $V \subseteq U$; we must show that $x, V \models_{\mathcal{T}} \psi$. By Proposition 6 (b), there is some $V^{\prime} \subseteq U$ in $\mathcal{F}$ with $V \in \operatorname{Rem}^{\mathcal{F}} V^{\prime}$. So $x, V^{\prime} \models_{\mathcal{F}} \psi$, and by induction hypothesis, $x, V^{\prime} \models_{\mathcal{T}} \psi$. By stability, $x, V \models_{\mathcal{T}} \psi$ also.

The other direction (if $x, U \models_{\mathcal{T}} \square \psi$, then $x, U \models_{\mathcal{F}} \square \psi$ ), is an easy application of the induction hypothesis.

Constructing the quotient of $\mathcal{T}$ under $\sim_{\mathcal{F}}$ is not adequate for generating a finite model because there may still be an infinite number of points. It turns out that we only need a finite number of them.

Let $\mathcal{M}=\langle X, \mathcal{T}, i\rangle$ be a topological model, and define an equivalence relation $\sim$ on $X$ by $x \sim y$ iff

(a) for all $U \in \mathcal{T}, x \in U$ iff $y \in U$, and

(b) for all atomic $A, x \in i(A)$ iff $y \in i(A)$.

Further, denote by $x^{*}$ the equivalence class of $x$, and let $X^{*}=\left\{x^{*} \mid x \in X\right\}$. For every $U \in \mathcal{T}$ let $U^{*}=\left\{x^{*} \mid x \in X\right\}$, then $\mathcal{T}^{*}=\left\{U^{*} \mid U \in \mathcal{T}\right\}$ is a topology on $X^{*}$. Define a map $i^{*}$ from the atomic formulae to the powerset of $X^{*}$ by $i^{*}(A)=\left\{x^{*} \mid x \in i(A)\right\}$. The entire model $\mathcal{M}$ lifts to the model $\mathcal{M}^{*}=\left\langle X^{*}, \mathcal{T}^{*}, i^{*}\right\rangle$ in a well-defined way.

Lemma 21 For all $x, U$, and $\phi$,

$$
x, U \models_{\mathcal{M}} \phi \quad \text { iff } \quad x^{*}, U^{*} \models_{\mathcal{M}^{*}} \phi .
$$

Proof. By induction on $\phi$.

Theorem 22 If $\phi$ is satisfied in any topological space then $\phi$ is satisfied in a finite topological space.

Proof. Let $\mathcal{M}=\langle X, \mathcal{T}, i\rangle$ be such that for some $x \in U \in \mathcal{T}, x, U \models_{\mathcal{M}} \phi$. Let $\mathcal{F}^{\phi}$ be a finite stable splitting (by Theorem 15) for $\phi$ and its subformulae with respect to $\mathcal{M}$. By Proposition 20, $x, U \models_{\mathcal{N}} \phi$, where $\mathcal{N}=\langle X, \mathcal{F}, i\rangle$. We may assume that $\mathcal{F}$ is a topology, and we may also assume that the overall language has only the (finitely many) atomic symbols which occur in $\phi$. Then the relation $\sim$ has only finitely many classes. So the model $\mathcal{N}^{*}$ is finite. Finally, by Lemma 21, $x^{*}, U^{*} \models_{\mathcal{N}^{*}} \phi$.

Observe that the finite topological space is a quotient of the initial one under two equivalences. The one equivalence is $\sim_{\mathcal{F}}^{\phi}$ on the open subsets of the topological space, where $\mathcal{F}^{\phi}$ is the finite splitting corresponding to $\phi$ and its cardinality is a function of the complexity of $\phi$. The other equivalence is $\sim_{X}$ on the points of the topological space and its number of equivalence classes is a function of the atomic formulae appearing in $\phi$. The following simple example shows how a topology is formed with the quotient under these two equivalences 
Example 23 Let $X$ be the interval $[0,1)$ of real line with the the set

$$
\mathcal{T}=\{\emptyset\} \cup\left\{\left[0, \frac{1}{2^{n}}\right) \mid n=0,1,2, \ldots\right\}
$$

as topology. Suppose that we have only one atomic formula, call it $A$, such that $i(A)=\{0\}$, then it is easy to see that the model $\langle X, \mathcal{T}, i\rangle$ is equivalent to the finite topological model $\left\langle X^{*}, \mathcal{T}^{*}, i^{*}\right\rangle$, where

$$
\begin{aligned}
X^{*} & =\left\{x_{1}, x_{2}\right\}, \\
\mathcal{T}^{*} & =\left\{\emptyset,\left\{x_{1}, x_{2}\right\}\right\}, \text { and } \\
i(A) & =\left\{x_{1}\right\} .
\end{aligned}
$$

So the overall size of the (finite) topological space is bounded by a function of the complexity of $\phi$. Thus if we want to test if a given formula is invalid we have a finite number of finite topological spaces where we have to test its validity. Thus we have the following

Theorem 24 The theory of topological spaces is decidable.

Observe that the last two results apply for lattices of subsets by Theorem 19 . Acknowledgements: I wish to thank Larry Moss and Rohit Parikh for helpful comments and suggestions.

\section{References}

[1991] Fagin, R., J. Y. Halpern, and M. Y. Vardi. A model-theoretic analysis of knowledge. Journal of the Association for Computing Machinery, 38(2):382428, 1991.

[1993] Georgatos, K. Modal logics for topological spaces. Ph.D. Dissertation. City University of New York, 1993.

[1980] Gierz, G., K. H. Hoffman, K. Keimel, J. D. Lawson, M. W. Mislove, and D. S. Scott. A Compendium of Continuous Lattices. Springer-Verlag, Berlin, Heidelberg, 1980.

[1984] Halpern, J. Y., and Y. Moses. Knowledge and common knowledge in a distributed environment. In Proceedings of the Third ACM Symposium on Principles of Distributed Computing, pages 50-61, 1984.

[1962] Hintikka, J. Knowledge and Belief. Cornell University Press, Ithaca, New York, 1962.

[1992] Moss, L. S., and R. Parikh. Topological reasoning and the logic of knowledge. In Y. Moses, editor, Proceedings of the Fourth Conference (TARK 1992), pages 95-105, 1992. 
[1985] Parikh, R., and R. Ramanujam. Distributed computing and the logic of knowledge. In R. Parikh, editor, Logics of Programs, number 193 in Lecture Notes in Computer Science, pages 256-268, Berlin, New York, 1985. Springer-Verlag.

[1968] Rasiowa, H., and R. Sikorski. The Mathematics of Metamathematics. Panstwowe Wydawnictwo Naukowe, Warszawa, Poland, second edition, 1968.

[1989] Vickers, S. Topology via Logic. Cambridge Studies in Advanced Computer Science. Cambridge University Press, Cambridge, 1989. 